\title{
Decay heat uncertainty quantification of MYRRHA
}

\author{
Luca Fiorito $^{1,2, a}$, Oliver Buss ${ }^{3, b}$, Axel Hoefer $^{3}$, Alexey Stankovskiy $^{1}$, and Gert Van den Eynde ${ }^{1}$ \\ ${ }^{1}$ SCK $\bullet$ CEN, Boeretang 200, 2400 Mol, Belgium \\ 2 ULB, Université Libre de Bruxelles, Avenue Franklin Roosevelt 50, 1050 Brussels, Belgium \\ 3 AREVA GmbH, Dept. Radiology \& Criticality, Kaiserleistrasse 29, 63067 Offenbach am Main, Germany
}

\begin{abstract}
MYRRHA is a lead-bismuth cooled MOX-fueled accelerator driven system (ADS) currently in the design phase at $\mathrm{SCK} \cdot \mathrm{CEN}$ in Belgium. The correct evaluation of the decay heat and of its uncertainty level is very important for the safety demonstration of the reactor. In the first part of this work we assessed the decay heat released by the MYRRHA core using the ALEPH-2 burnup code.

The second part of the study focused on the nuclear data uncertainty and covariance propagation to the MYRRHA decay heat. Radioactive decay data, independent fission yield and cross section uncertainties/covariances were propagated using two nuclear data sampling codes, namely NUDUNA and SANDY. According to the results, ${ }^{238} \mathrm{U}$ cross sections and fission yield data are the largest contributors to the MYRRHA decay heat uncertainty. The calculated uncertainty values are deemed acceptable from the safety point of view as they are well within the available regulatory limits.
\end{abstract}

\section{Introduction}

MYRRHA [1] is a multi-purpose irradiation facility conceived as an accelerator-driven system (ADS) and currently in design phase at $\mathrm{SCK} \cdot \mathrm{CEN}$. MYRRHA is fueled with MOX, cooled down by liquid eutectic $\mathrm{Pb}-\mathrm{Bi}$ (LBE) and is supposed to operate in both critical and subcritical mode. In the latter case, the MYRRHA linear accelerator delivers a continuous wave $600 \mathrm{MeV}$ proton beam to the LBE spallation target, which acts as a neutron source for the subcritical core.

In the reactor design phase, a primary role was assigned to the evaluation of the MYRRHA fuel decay heat, i.e., the power generated by the fuel after the reactor shut-down. Depending on the system under study, it can reach several percent of the operational nominal power.

The instantaneous decay heat $D H_{i}(t)$ released at time $t=T$ by a nuclide with isotopic density $n_{i}(T)$ is described as the average decay energy $E_{i}^{d}$ released by one disintegration multiplied by the decay rate $\lambda_{i} n_{i}(T)$ of the nuclide concentration in the system. The sum of the decay heat produced by each isotopic component of a nuclear material gives the total decay heat for that material: $D H(T)=\sum_{i} \lambda_{i} n_{i}(T) E_{i}^{d}$. The reliability of this calculation comes from the accuracy of the computational model and nuclear data, along with an estimate of the uncertainty bounds. The latter determines the operational range of the safety systems dedicated to the decay heat removal, which prevent the overheating and mechanical failure of the reactor components, e.g. structure bending, cladding and fuel failure.

In this work the decay heat of the subcritical MYRRHA core was calculated up to several thousands of years of decay using the Monte Carlo burnup code

\footnotetext{
a e-mail: Ifiorito@sckcen.be

b e-mail: oliver.buss@areva.com
}

ALEPH-2 [2]. The decay heat was apportioned to fuel batches with different burnup levels and the nuclides producing the largest contribution were identified. Then, the uncertainties/covariances of decay constants, decay energies, independent fission yields and cross sections were propagated through the model and the decay heat uncertainty was quantified. NUDUNA [3] and SANDY [4] were the codes selected for the nuclear data uncertainty propagation. From the analysis of the results we were able to assess which nuclear data introduce the largest uncertainties on the decay heat evaluation.

\section{Decay heat calculation for the MYRRHA core}

\subsection{MYRRHA decay heat model}

The decay heat calculation was performed on the equilibrium, beginning-of-cycle (BOC) sub-critical core of MYRRHA, which consists of 72 fuel assemblies grouped in twelve batches of six according to the symmetry in the neutron flux distribution [5]. The central sub-assembly (SA) hosts the beam tube and the beam window and it is filled by LBE. Six in-pile sections (IPS) surround the spallation target at the second radial layer, six SAs contain safety rods and six SAs are dedicated to the radioisotope production. The reflector assemblies are 44 . The top view at the core mid-plane is shown in Fig. 1.

The fuel type is a $30 \mathrm{wt} . \%(\mathrm{Pu}+\mathrm{Am}) \mathrm{MOX}$ [6] burned to different burnup levels. Batch 1 and 12 contain respectively fresh fuel and the most irradiated fuel at BOC with a cumulative burnup of $55.6 \mathrm{MWd} / \mathrm{kg}_{\text {HM }}$ [5]. Details on the fuel batches are reported in Table 1.

The fuel was irradiated for one cycle of 90 days, in which the power level was maintained constant at 70 MW. Decay followed for all fuel batches up to several

(C) The Authors, published by EDP Sciences. This is an Open Access article distributed under the terms of the Creative Commons Attribution License 4.0 (http://creativecommons.org/licenses/by/4.0/). 


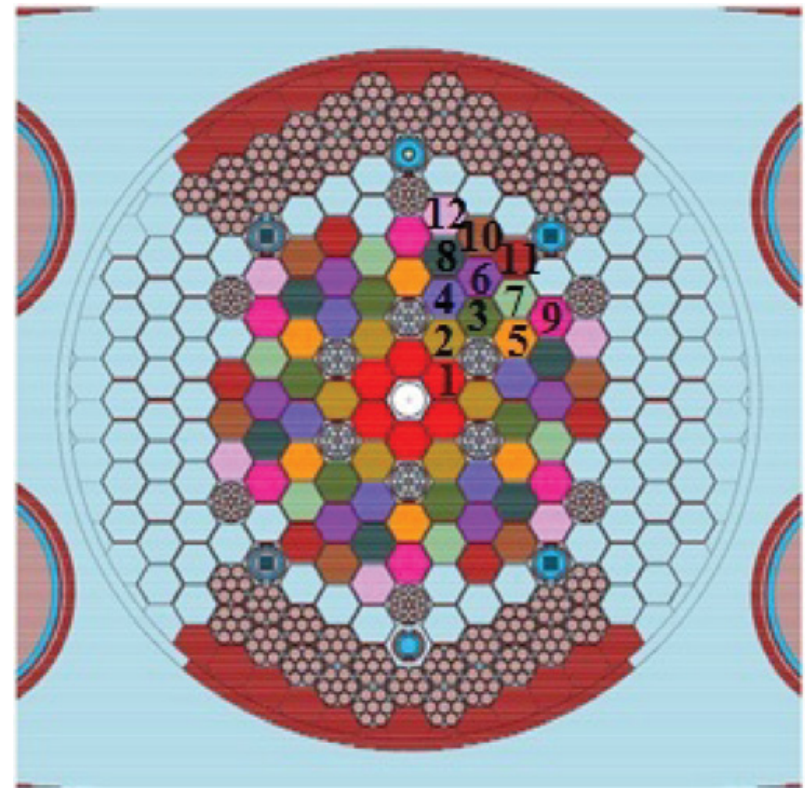

Figure 1. MYRRHA sub-critical BOC core layout. Twelve fuel batches are shown in different colors and are numbered according to the descending level of initial burnup at BOC.

Table 1. Details of the MYRRHA subcritical core fuel batches.

\begin{tabular}{cccc}
\hline $\begin{array}{c}\text { batch } \\
\#\end{array}$ & $\begin{array}{c}\text { BU at BOC } \\
\left(\mathrm{MWd} / \mathrm{kg}_{\mathrm{HM}}\right)\end{array}$ & $\begin{array}{c}\text { DH at EOC } \\
(\mathrm{MW})\end{array}$ & $\begin{array}{c}\mathrm{P}_{t h}=\text { const } \\
(\mathrm{MW})\end{array}$ \\
\hline 1 & 0 & 0.51 & 9.29 \\
2 & 7.78 & 0.46 & 8.12 \\
3 & 14.65 & 0.45 & 7.92 \\
4 & 21.39 & 0.36 & 6.19 \\
5 & 26.57 & 0.32 & 5.54 \\
6 & 31.3 & 0.31 & 5.33 \\
7 & 35.81 & 0.29 & 5.06 \\
8 & 40.1 & 0.29 & 5.05 \\
9 & 44.37 & 0.26 & 4.54 \\
10 & 48.2 & 0.26 & 4.41 \\
11 & 51.9 & 0.25 & 4.36 \\
12 & 55.6 & 0.24 & 4.18 \\
\hline Total & \multicolumn{4}{c}{} \\
\hline \hline
\end{tabular}

thousands of years. The MYRRHA decay heat calculation was modelled with the ALEPH-2 code [2].

\subsection{Decay heat results}

The decay heat profiles for the twelve fuel batches calculated with ALEPH-2 using the JEFF-3.1.2 [7] nuclear data library are reported in Fig. 2.

The fresh fuel in batch 1 generates the largest amount of decay heat, especially at short decay times (some days). However, in normal operation only batch 12 is removed from the reactor core and let decay. Batches from 1 to 11 are shuffled in the core and new fresh fuel is loaded in the previous position of batch 1 . The list of nuclides that individually contribute to more than $1 \%$ of the decay heat produced by batch 12 is reported in Figs. 3 and 4.

Actinides ${ }^{239} \mathrm{U},{ }^{239} \mathrm{~Np}$ and ${ }^{242} \mathrm{Cm}$ and fission products ${ }^{134} \mathrm{I},{ }^{104,106} \mathrm{Tc},{ }^{138,140} \mathrm{Cs},{ }^{100} \mathrm{Nb}$ and ${ }^{140,144} \mathrm{La}$ are amongst the largest contributors to the decay heat of the MYRRHA fuel up to few days of decay. Afterwards only actinides play a role, i.e., ${ }^{242} \mathrm{Cm},{ }^{238,240,239} \mathrm{Pu}$ and ${ }^{242} \mathrm{Am}$.

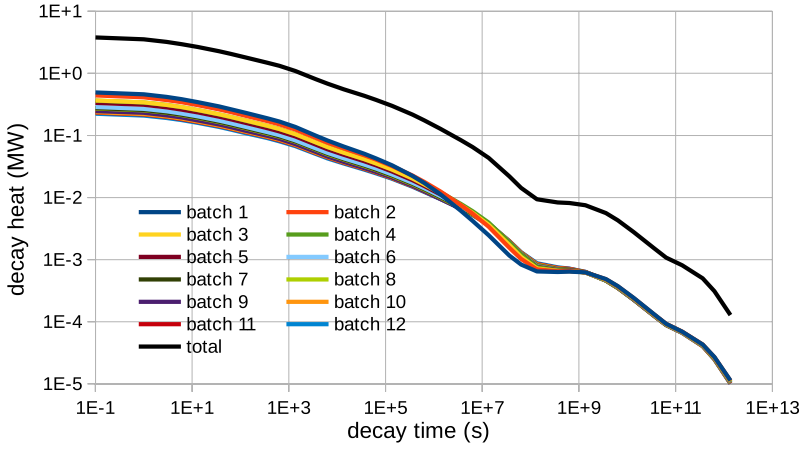

Figure 2. Decay heat profile apportioned to the twelve fuel batches of the MYRRHA subcritical core.

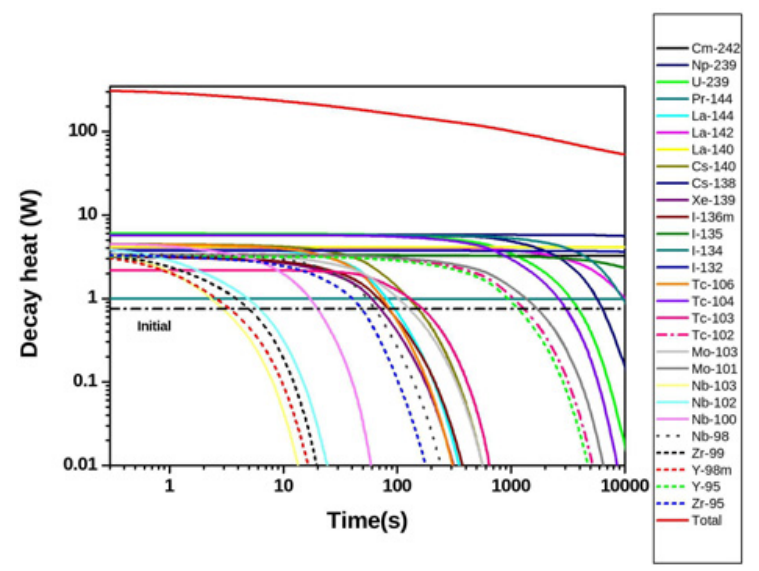

Figure 3. Nuclides that contribute the most to the decay heat released by batch 12 of the MYRRHA fuel. Cooling time up to $1 \times 10^{4}$ seconds. The curve "initial" refers to the energy released by fresh fuel.

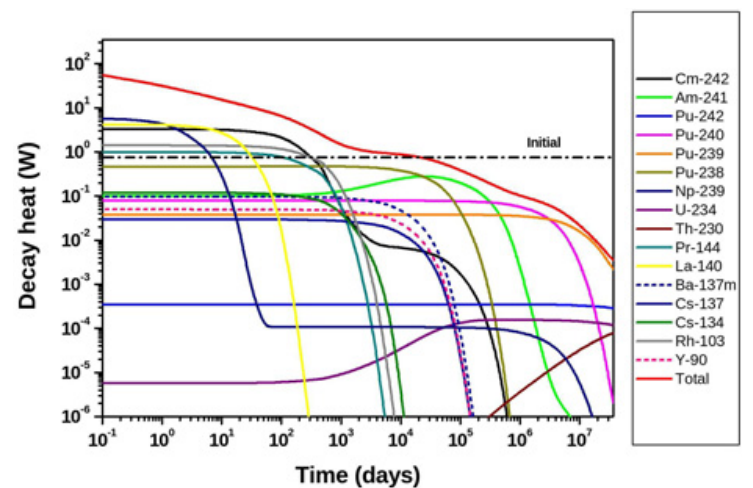

Figure 4. Nuclides that contribute the most to the decay heat released by batch 12 of the MYRRHA fuel. Cooling time between $10^{4}$ seconds and $10^{5}$ years. The curve "initial" refers to the energy released by fresh fuel.

\section{Decay heat uncertainty quantification}

The uncertainty propagation was carried out with the NUDUNA code [3] for the cross section data and with the SANDY code [4] for fission yields, decay constants and energies. Both codes base the uncertainty propagation on the random sampling of the input nuclear data according to their uncertainty/covariance information. After running the model for each set of samples, one can calculate the uncertainty of the selected responses from the statistical analysis of the outputs. The main advantage of sampling 
Table 2. Details on NUDUNA and SANDY.

\begin{tabular}{|c|c|c|}
\hline & NUDUNA & SANDY \\
\hline institute: & AREVA GmbH & $\mathrm{SCK} \cdot \mathrm{CEN}$ \\
\hline input format: & & \\
\hline ENDF-6 & $\checkmark$ & $\checkmark$ \\
\hline can work with (ENDF-6 file): & & \\
\hline fission neutron multiplicities (file 31) & $\checkmark$ & $\checkmark$ \\
\hline resonance parameters (file 32) & $\checkmark$ & $\checkmark$ \\
\hline cross sections (file 33) & $\checkmark$ & $\checkmark$ \\
\hline angular distributions (file 34) & $\checkmark$ & $\checkmark$ \\
\hline energy distributions (file 35) & $x$ & $\checkmark$ \\
\hline radioactive decay data (file 8 ) & $\checkmark$ & $\checkmark$ \\
\hline fission yields (file 8) & $x$ & $\checkmark$ \\
\hline output format: & & \\
\hline ENDF-6 / PENDF & $\checkmark$ & $\checkmark$ \\
\hline $\mathrm{ACE}$ & $\checkmark$ & $\checkmark$ \\
\hline AMPX & $\checkmark$ & $x$ \\
\hline extra features: & & $\begin{array}{l}\text { - variance decomposition } \\
\text { - fission yield covariances }\end{array}$ \\
\hline
\end{tabular}

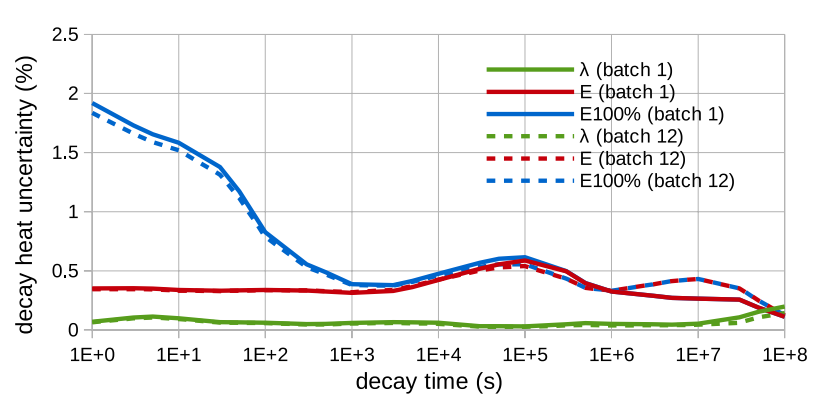

Figure 5. Decay heat uncertainty produced by radioactive decay data.

codes is their straightforward application to any model, as they do not interact with the model itself but only with its inputs. In addition, sampling methods do not introduce (first-order) model surrogates as an approximation and do not apply locally around the input best estimates but cover the whole input and output phase space, provided that enough samples are taken. Extra details on the two codes are reported in Table 2.

In this work, only the decay heat uncertainties of the batches with the lowest (batch 1) and highest (batch 12) burnup level at BOC are reported. Figure 5 shows the uncertainty profiles produced with the radioactive decay data sampled from JEFF-3.1.2 ${ }^{1}$, namely, the decay constants and the average decay energy values.

Decay constants have a negligible impact on the decay heat uncertainty. The uncertainty produced by the JEFF-3.1.2 decay energy data is lower than $0.5 \%$. However, these data are often incomplete, as more than 2000 decay energy values are given without an evaluated uncertainty. The results obtained with this assumption could seriously underestimate the uncertainty level of the decay heat. As proposed in [8] the decay heat uncertainty profiles were recalculated introducing an extreme $100 \%$ uncertainty where no evaluated decay energy uncertainty was found. With such a correction, the decay energy data contribute to a

\footnotetext{
${ }^{1}$ Updated neutron-induced data are available in JEFF-3.1.2 compared to the previous release JEFF-3.1.1. However, radioactive decay data and fission yields were not changed.
}

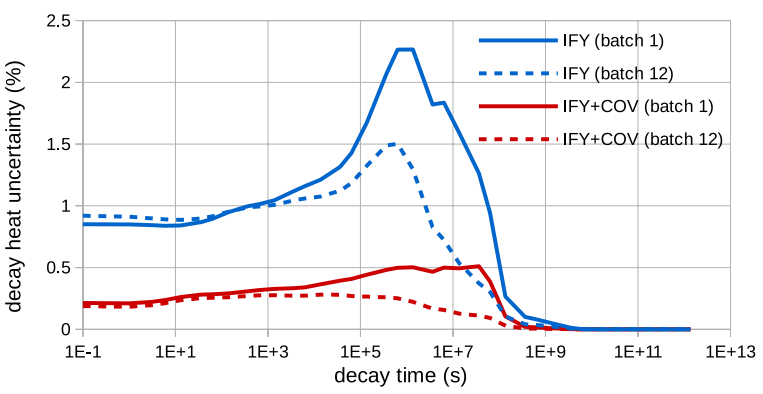

Figure 6. Percent of the MYRRHA decay heat uncertainty that can be associated with independent fission yield (IFY) uncertainties and covariances.

decay heat uncertainty larger than $1 \%$ for the first 100 seconds after shut-down.

Independent fission yields introduce the most of the uncertainty into the model ( $>2 \%$ at $10^{6}$ seconds), as reported in Fig. 6. Their impact is more evident for batch 1 where more fission events occur. The sudden rise in the uncertainty profile generated by the independent fission yields at $\approx 10^{6}$ seconds is associated to the accumulation of ${ }^{140} \mathrm{La}$, which is mostly produced by the radioactive decay of ${ }^{140} \mathrm{Xe},{ }^{140} \mathrm{Cs}$ and ${ }^{140} \mathrm{Ba}$. The large independent fission yield uncertainties of these three isotopes - i.e., respectively, $23.68 \%, 34.79 \%$ and $13.72 \%$ — are the origin of this behavior. The fission yield uncertainty contribution becomes less relevant for longer decay times, as the actinides produce most of the decay heat.

The JEFF-3.1.2 fission yield data do not contain covariance matrices. SANDY can generate such covariances using the generalized least-squares method described in [9], which adjusts the current data to the available knowledge of observables such as the chain fission yields [10]. Figure 6 shows that the impact of independent fission yield covariances on the decay heat uncertainty reduces by far the importance of such data.

The reaction cross section covariances of several nuclides were propagated using NUDUNA and the results are reported in Fig. 7. Because of the lack of covariance data in JEFF-3.1.2, samples were drawn using ENDF/BVII.1 [11]. Cross section data were propagated for those 


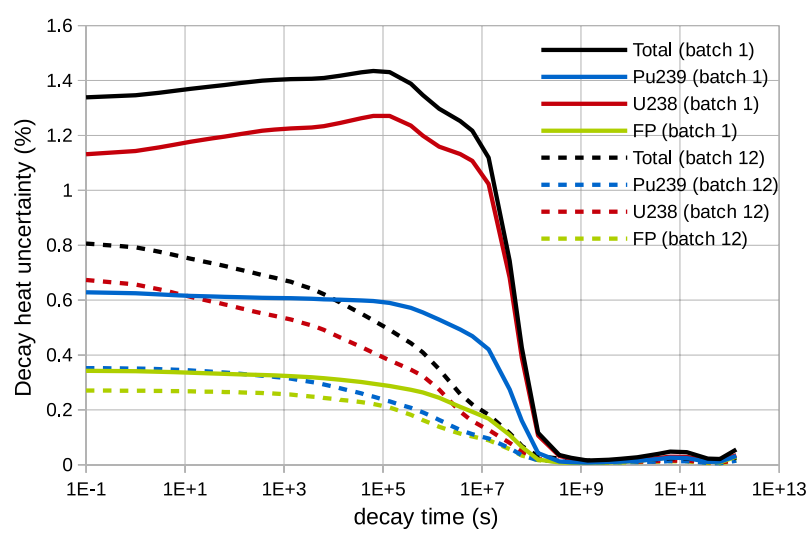

Figure 7. Percent of the MYRRHA decay heat uncertainty that can be associated with cross section covariance data. The cross section covariance contribution was grouped by nuclide: ${ }^{238} \mathrm{U}$, ${ }^{239} \mathrm{Pu}$ and fission products (FP).

nuclides important for the MYRRHA reactor operation, safety and radiological consequences: actinides $\left({ }^{238} \mathrm{U}\right.$, ${ }^{239} \mathrm{Pu}$ ), fission products $\left({ }^{99} \mathrm{Tc},{ }^{103} \mathrm{Rh},{ }^{106} \mathrm{Pd},{ }^{131,132} \mathrm{Xe}\right.$, $\left.{ }^{133,135} \mathrm{Cs},{ }^{143} \mathrm{Nd},{ }^{149,151,152} \mathrm{Sm},{ }^{153} \mathrm{Eu},{ }^{155,157} \mathrm{Gd}\right)$ and light nuclides $\left({ }^{1} \mathrm{H},{ }^{10} \mathrm{~B}\right)$. Cross sections introduce the largest decay heat uncertainty contribution of batch 1 at short and intermediate decay times. This effect drops to inappreciable levels after $10^{7}$ seconds, when the actinides generate most of the decay heat.

Then, the cross sections uncertainty contribution was decomposed by nuclide. Since fission products contribute to the decay heat up to a few days of decay, the cross section covariances of ${ }^{239} \mathrm{Pu}$ - where approximatively $70 \%$ of the fission events occur- largely affect their concentrations and produce an uncertainty on the decay heat for that time interval. This contribution is around $0.6 \%$ for the fresh fuel in batch 1 , which refers to $\approx 3 \mathrm{~kW}$ of decay heat uncertainty immediately after shut down. ${ }^{238} \mathrm{U}$ cross section data produce more than $1 \%$ of uncertainty on the decay heat as long as the concentrations of ${ }^{239} \mathrm{U}$ and ${ }^{239} \mathrm{~Np}$ are significant. The uncertainty generated by fission products and light elements' cross section covariances is close to the statistical errors of the sampling and should be considered negligible.

\section{Conclusions}

The decay heat profile and its uncertainty associated to the nuclear data were calculated for the MYRRHA subcritical core and apportioned to 12 batches of fuel with different burnup level. It was assessed that ${ }^{239} \mathrm{U},{ }^{239} \mathrm{~Np}$ and fission products contribute significantly to the decay heat production up to a few days. Afterwards, only actinides play individually a significant role.

Two sampling codes, i.e., NUDUNA and SANDY, were used to propagate the nuclear data uncertainties and covariances of radioactive decay data, cross sections and fission yields to the decay heat. The calculated uncertainty remains always below a few percent for each fuel batch and becomes negligible after a few years of decay. The uncertainty's largest contributors were identified in the ${ }^{238} \mathrm{U}$ cross sections. Also, several independent fission yields have a major effect, especially for the production of ${ }^{140} \mathrm{La}$, which accounts for $\approx 30 \%$ of decay heat after a few days. This effect can be reduced by introducing physical covariances for independent fission yields.

The uncertainty produced on the MYRRHA decay heat by the current nuclear data are lower than the value provided by the regulatory guides for traditional thermal reactors. However, considering that decay heat measurements for fast reactors or analogous systems are few (fission pulses) or not existing, extra care is recommended in the application of these results in the reactor design phase. On the other hand, the attention should be drawn on the generation of the nuclear data uncertainty and its predictive role for systems such as MYRRHA.

\section{References}

[1] H. Ait Abderrahim, P. Baeten, D. De Bruyn, R. Fernandez, Energy conversion and management 63, 4 (2012)

[2] A. Stankovskiy, G. Van den Eynde, Science and Technology of Nuclear Installations 2012 (2012)

[3] C. Díez, O. Buss, A. Hoefer, D. Porsch, O. Cabellos, Annals of Nuclear Energy 77, 101 (2015)

[4] L. Fiorito, G. Žerovnik, A. Stankovskiy, G.V. den Eynde, P. Labeau, Submitted to Annals of Nuclear Energy (2016)

[5] E. Malambu, A. Stankovskiy, Tech. Rep. SCK•CEN/3958903, SCK•CEN (2014)

[6] V. Sobolev et al., Tech. Rep. SCK•CEN-R-5151, SCK•CEN (2011)

[7] A. Santamarina, D. Bernard, P. Blaise, M. Coste, A. Courcelle, T. Huynh, C. Jouanne, P. Leconte, O. Litaize, S. Mengelle et al., Tech. Rep. JEFF report 22, OECD/NEA Data Bank (2009)

[8] J. Katakura, Journal of Nuclear Science and Technology 50(8), 799 (2013)

[9] L. Fiorito, A. Stankovskiy, G.V. den Eynde, C. Diez, O. Cabellos, P. Labeau, Annals of Nuclear Energy 88, 12 (2016), ISSN 0306-4549, http://www.sciencedirect.com/science/ article/pii/S0306454915005137

[10] A. Nichols, D.L., Aldama, M. Verpelli, Tech. Rep. INDC(NDS)-0534, IAEA (2008)

[11] M. Chadwick, M. Herman, P. Oblozinsky, M. Dunn, Y. Danon, A. Kahler, D. Smith, B. Pritychenko, G. Arbanas, R. Arcilla et al., Nuclear Data Sheets 112(12), 2887 (2011) 